\title{
Correlations and Event-by-Event Fluctuations in High Multiplicity Events Produced in ${ }^{208} \mathbf{P b}-{ }^{208} \mathrm{~Pb}$ Collisions
}

\author{
Shakeel Ahmad (D), ${ }^{1}$ Shaista Khan, ${ }^{1}$ Ashwini Kumar (D), ${ }^{2}$ \\ Arpit Singh, ${ }^{3}$ A. Ahmad, ${ }^{4}$ and B. K. Singh $~^{1}{ }^{3}$ \\ ${ }^{1}$ Department of Physics, Aligarh Muslim University, Aligarh 202002, India \\ ${ }^{2}$ Department of Physics and Electronics, Dr. Ram Manohar Lohia Avadh University, Faizabad 224001, India \\ ${ }^{3}$ Department of Physics, Banaras Hindu University, Varanasi 221005, India \\ ${ }^{4}$ Department of Applied Physics, Aligarh Muslim University, Aligarh 202002, India
}

Correspondence should be addressed to Shakeel Ahmad; shakeel.ahmad@cern.ch

Received 27 October 2017; Accepted 7 March 2018; Published 8 April 2018

Academic Editor: Andrea Coccaro

Copyright (C) 2018 Shakeel Ahmad et al. This is an open access article distributed under the Creative Commons Attribution License, which permits unrestricted use, distribution, and reproduction in any medium, provided the original work is properly cited. The publication of this article was funded by SCOAP

\begin{abstract}
Analysis of high multiplicity events produced in $158 \mathrm{~A} \mathrm{GeV} / \mathrm{c}^{208} \mathrm{~Pb}-{ }^{208} \mathrm{~Pb}$ collisions is carried out to study the event-by-event fluctuations. The findings reveal that the method of scaled factorial moments can be used to identify the events having densely populated narrow phase space bins. A few events sorted out by adopting this approach are individually analyzed. It is observed that these events do exhibit large fluctuations in their pseudorapidity, $\eta$, and azimuthal angle, $\phi$, distributions arising due to some dynamical reasons. Two-particle $\Delta \eta-\Delta \phi$ correlation study applied to these events too indicates that some complex two-dimensional structure of significantly high magnitude is present in these events which might have some dynamical origin. The findings reveal that the method of scaled factorial moments may be used as an effective triggering for events with large dynamical fluctuations.
\end{abstract}

\section{Introduction}

Investigations involving fluctuations in collisions of heavy nuclei at relativistic energies might serve as a useful tool to identify the existence of the state of partonic matter in early life of the fireball because of the idea that the fluctuations in a thermal system are directly related to various susceptibilities and would be a good indication for the possible phase changes [1-4]. Furthermore, large event-byevent (ebe) fluctuations of the suitably chosen observables in ion-ion (AA) collisions would help in identifying events of distinct classes, for example, one with and another without quark-gluon plasma (QGP) [2], as, under extreme conditions of temperature and energy density, a novel phase of matter, the QGP, is expected to be produced. The search for the occurrence of phase transition from hadronic matter to QGP still remains a favorite topic for high energy physicists $[1$, $5,6]$. It is commonly believed that even if the extreme conditions of QGP formation are achieved in relativistic AA collisions, not all the events would be produced via QGP as the cross section for the QGP formation [1] is still not known. Therefore, a chosen subsample of events exhibiting large fluctuations in certain observables should be studied in detail $[7,8]$. A significant contribution to the observed fluctuations in a variable comes due to finite number of particles produced in an event and is referred to as the statistical fluctuations. Its magnitude could be evaluated by considering the independent emission of particles or by using the event mixing technique $[1,2,7,8]$. All other fluctuations are of dynamical origin and are divided into two groups: (a) fluctuations which do not change on ebe basis, for example, two-particle correlation due to Bose-Einstein statistics or due to decay of resonances and (b) fluctuations which change on ebe basis and are termed as ebe fluctuations. Examples are fluctuations in charged to neutral particle multiplicity ratio due to creation of disoriented chiral condensate (DCC) region or creation of jets which contribute to the high $p_{t}$ tail of transverse momentum distributions $[1,2]$. Several attempts 
[1-3, 9-14] have been made to investigate ebe fluctuations in heavy-ion collisions at relativistic energies. The findings do indicate the presence of dynamical fluctuations. The main aim of the present study is to search for the rare events exhibiting some unusual behaviour from a data sample with large number of events. The analyses of individual collision events at SPS and RHIC energies have been argued to statistically reliable as the multiplicities of these events would be high enough and the statistical fluctuations may be treated as under control [15-22]. Moreover, from the study of such events with strong fluctuations, information about the dominance of dynamical components of fluctuations over the statistical ones can be extracted, which, in turn, would provide more insight into the underlying dynamics of high multiplicity events [23, 24]. Analyses of one "hadron-rich" event [25] in the context of Centauro event, single event $p_{t}$ distributions [26], single event $k / \pi$ ratio [27], intermittency in individual events, entropy and cluster analysis in single events $[21,22]$, and so on are some of the investigations carried out so far.

An attempt, therefore, has been made to carry out the analysis of a few high multiplicity individual events produced in $158 \mathrm{~A} \mathrm{GeV} / \mathrm{c} \mathrm{Pb}-\mathrm{Pb}$ collisions. These events are taken from the emulsion experiment performed by EMU01 Collaboration [28-31]. It should be emphasized that the conventional emulsion technique has an advantage over the other detectors due to its $4 \pi$ solid angle coverage and the data are free from biases due to full phase space acceptance whereas other detectors have limited acceptance cone. This not only reduces the charged particle multiplicity but might also distort some of the event characteristics, like particle density fluctuations. Furthermore, to test whether the fluctuations are arising due to nonstatistical reasons, the findings are compared with the reference distributions obtained by event mixing technique $[15,16,32]$.

\section{Results and Discussion}

2.1. Single Event Factorial Moments. Method of scaled factorial moments (SFMs) [33-35] has been extensively used $[1,15,36]$ to search for the nonlinear phenomena in hadronic and ion-ion collisions at widely different energies. The power law behaviour of the type $F_{q} \sim M_{q}^{\psi}$ has been investigated by studying $[37,38]$ the horizontally averaged vertical moments $F_{q}^{v}$ and (or) the vertically averaged horizontal moments, $F_{q}^{h}$. The results suggest the presence of large particle density fluctuations in narrow phase space bins to be rare that cannot be ignored. It has, however, been pointed out [37, 39] that because of the averaging procedure adopted, studies involving $F_{q}^{v}$ or $F_{q}^{h}$ may not fully account for all the fluctuations a system might exhibit. Moreover, the values of the factorial moments $F_{q}^{e}$, estimated on ebe basis, have been observed to exhibit large fluctuations and hence a distinct distribution of $F_{q}^{e}$ for a given order $q$ and bin width, $\delta$, may be obtained for a given data sample $[18,37,39]$. This result suggests that the method of SFMs may be applied to individual events $[15,40,41]$ to search for the nonlinear phenomena in "hot" and "cold" events; the "hot" and "cold" events refer to the events, respectively, with and without large particle densities in narrow phase space bins. Method of SFMs applied to individual high multiplicity cosmic ray events [41] does indicate the presence of dynamical fluctuations. Such analysis, if carried out on ebe basis would help selecting in the events with large dynamical fluctuations, if this property is not a typical one for each single event. These suppositions have, therefore, been tested in the present study by applying the SFMs analysis to the individual events.

The event factorial moments of order $q$ are defined as

$$
F_{q}^{e}=\frac{\langle n(n-1) \cdots(n-q+1)\rangle_{e}}{\langle n\rangle_{e}^{q}},
$$

where $n$ denotes the multiplicity in a particular $\eta$ or $\phi$ bin.

Values of $F_{q}^{e}$ for $q=2$ are calculated for each of 47 events considered by varying the number of cells, $M$. Since the number of events are limited, instead of plotting the $F_{2}^{e}$ distributions; we have plotted event-wise $\ln F_{2}$ values for $M=$ 10 and 30 (in the pseudorapidity $(\eta=-\ln \tan \theta / 2)$ and the azimuthal angle $(\phi)$ spaces), with $\theta$ being the emission angle of a charged particle with respect to the mean beam direction. For the values $M=25$ and 100, we have shown the plot in two-dimensional $\eta-\phi$ space. These plots are shown in Figure 1. Values of $\ln F_{2}$ for the corresponding mixed events are also displayed in the same figure for comparison sake. It may be noted from the figure that $\ln F_{2}$ values of mixed events are nearly the same $\sim 0.3$ whereas these values for the real data vary from event to event such that one would get a distinct distribution of $\ln F_{2}$ for a significant number of events. It is quite interesting to notice that the values of $\ln F_{2}$ for few of the real events are much higher as compared to the average values taken over all the events or the values from the mixed events. These observations, thus, help in identifying a few events exhibiting significantly large $F_{2}$ values in $\eta^{-}, \phi$-, or $\eta-\phi$ space for further analysis. Four such events picked-up are labeled as Evt\# 5, 16, 21, and 27. These events henceforth, would be termed as "hot" events. The multiplicities of these events are, respectively, 932, 852, 1433, and 974. Yet another event, Evt\# 22 , having $F_{2}$ values close to the mixed events values has also been taken for comparison sake. This event henceforth will be referred to as the "cold" event. The multiplicity of this event is 1480. Variations of $\ln F_{2}$ with $\ln M$ for these five real and corresponding mixed events in $\eta^{-}, \phi$-, or $\eta^{-}$ $\phi$ spaces are displayed in Figure 2. Event averaged values, $\left\langle\ln F_{2}\right\rangle$ against $\ln M$ for the entire event sample are also plotted in the same figure. It is evidently clear from Figure 2 that $F_{2}$ values for the four "hot" events are significantly larger than that of the "cold" event or the event averaged values. These findings, therefore, tend to suggest a few events with high density phase region or strong fluctuations are present in the real data and that these fluctuations might have some dynamical origin. These dynamical fluctuations are the reflection of the dynamics and response of the system and are indicative of phase transition like phenomenon occurring under extreme conditions of heavy-ion collision such as the conditions achieved here at $158 \mathrm{~A} \mathrm{GeV} / \mathrm{c}$. This result, in turn, leads us to conclude that the method of SFMs, if applied to the single event, may be useful for selecting the preliminary 

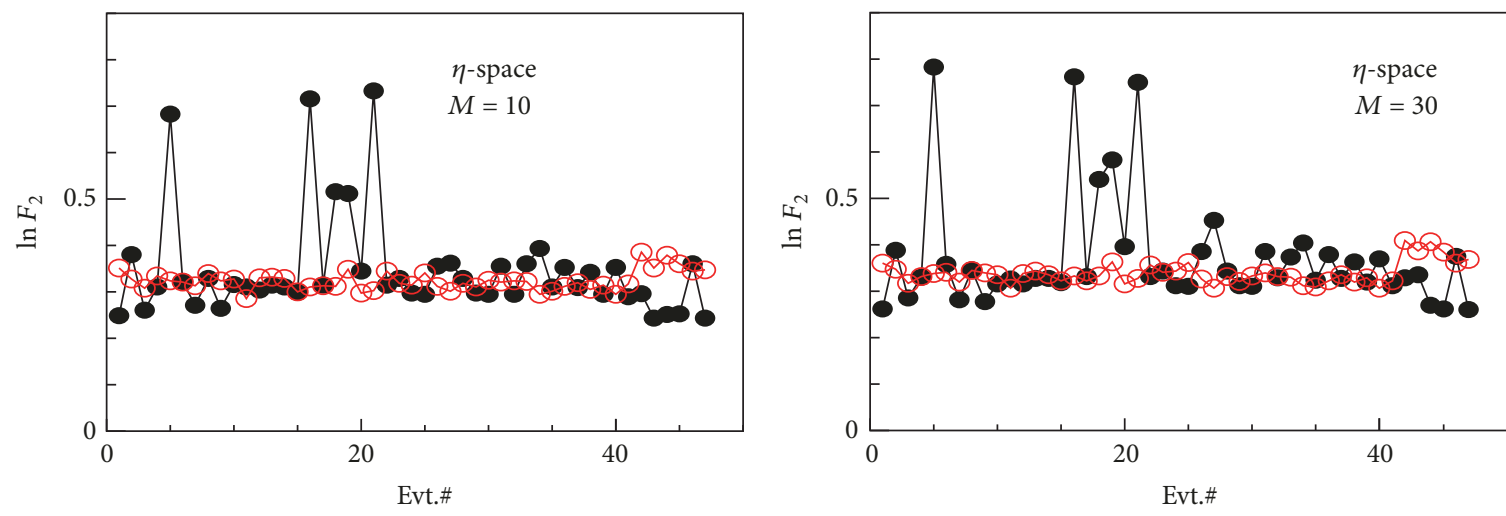

- Data

- Data

$\ominus$ Mixed

$\ominus$ Mixed

(a)
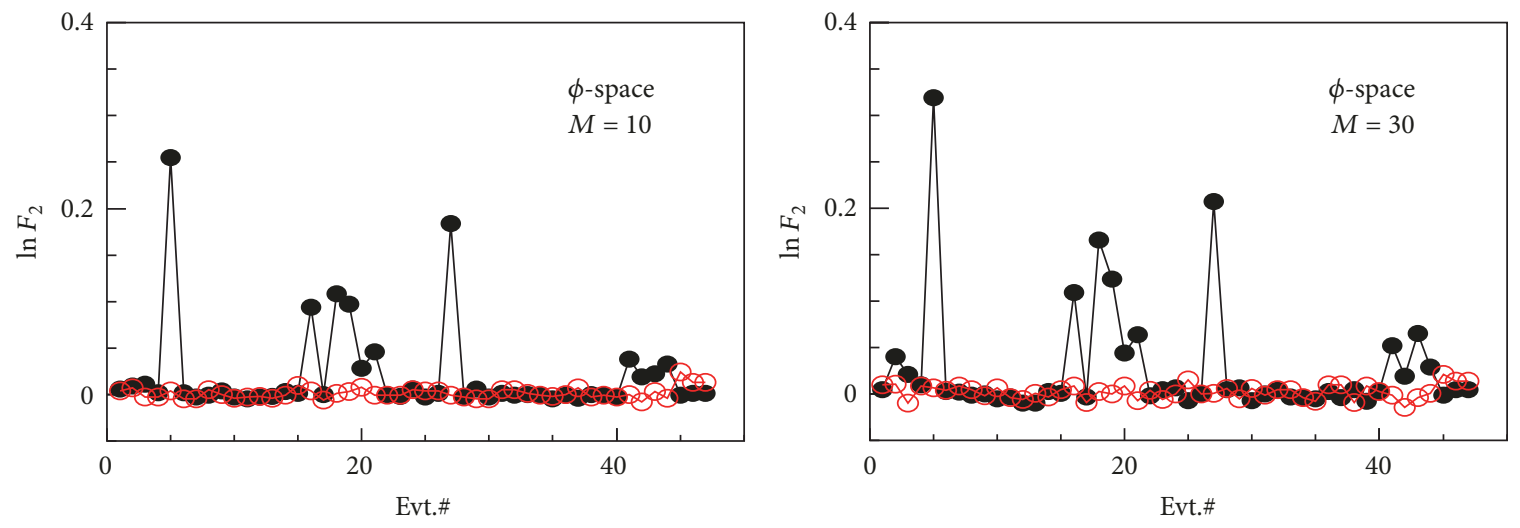

- Data

- Data

$\ominus$ Mixed

$\ominus$ Mixed

(b)
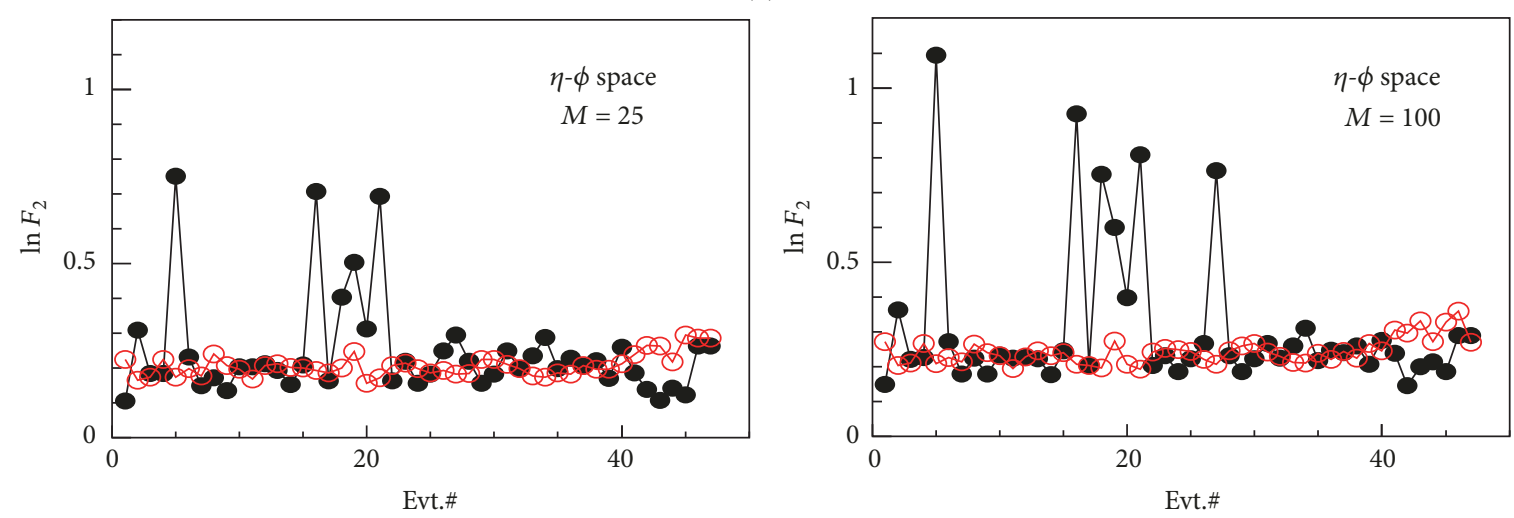

$$
\text { - Data }
$$

$\ominus$ Mixed

- Data

$\ominus$ Mixed

(c)

Figure 1: Event-wise variations of $\ln F_{2}$ in $\eta$-space (a), $\phi$-space (b), and $\eta-\phi$ space (c) for the data and mixed events produced in ${ }^{208} \mathrm{~Pb}-{ }^{208} \mathrm{~Pb}$ collisions at $158 \mathrm{~A} \mathrm{GeV} / \mathrm{c}$.

events where strong dynamical fluctuations might be present and thereafter more advanced triggering might occur; for example, particle ratios and enhanced particle multiplicities in certain kinematical regions may be applied.
2.2. $\eta$ and $\phi$ Distribution of Single Event. By examining the $\mathrm{F}_{2}$ values on ebe basis it might be possible to identify the rare events having high density phase space regions. Therefore, to check further that the four "hot" events identified as above 

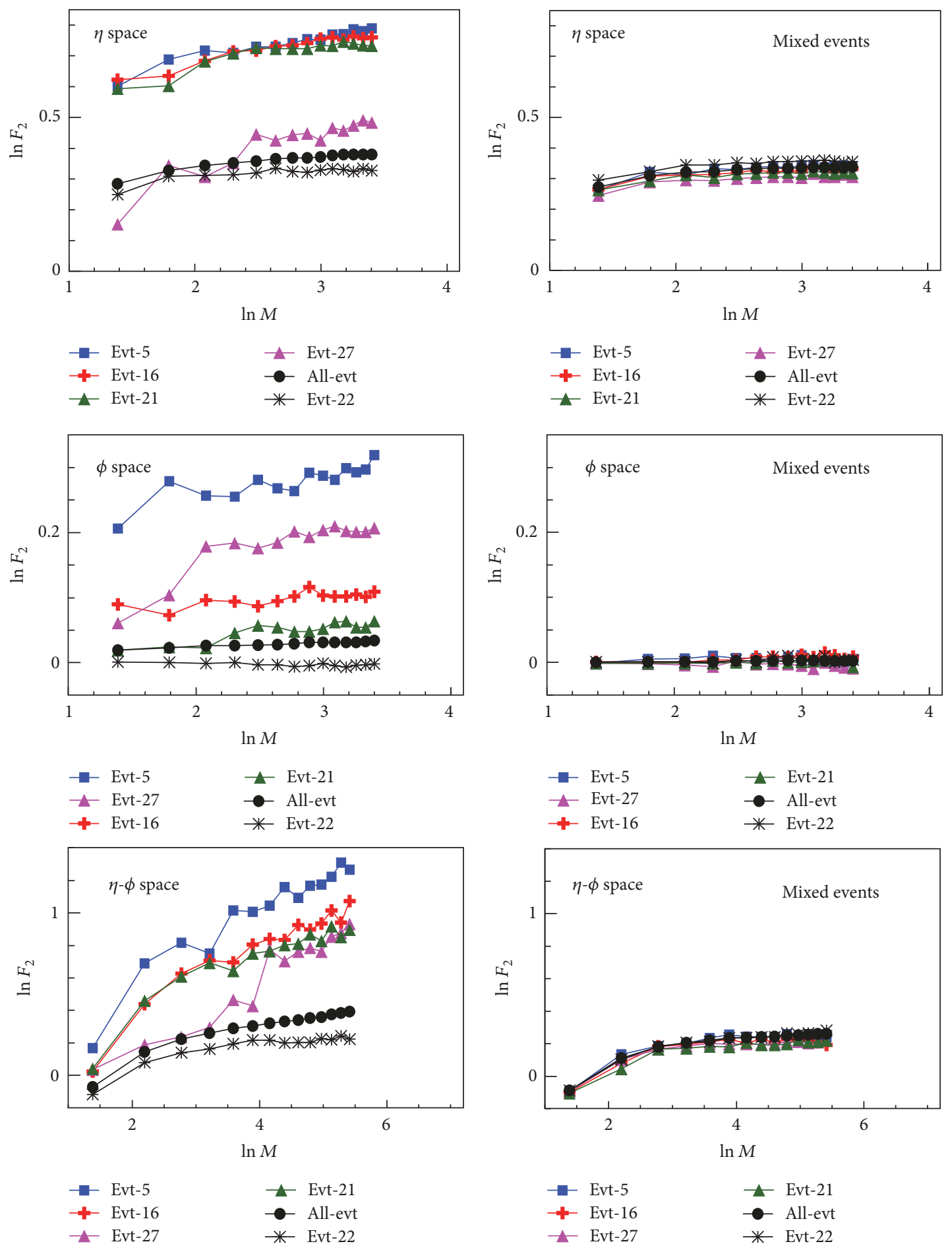

(a)
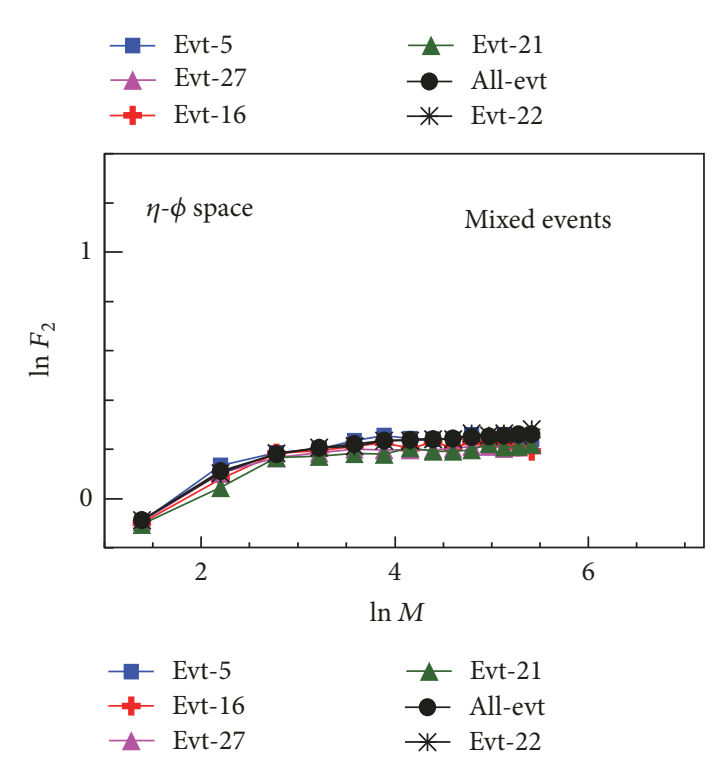

(b)

Figure 2: Variations of $\ln F_{2}$ with $\ln M$ in $\eta^{-}, \phi$-, and $\eta-\phi$ spaces for all and five individual events produced in $158 \mathrm{~A} \mathrm{GeV/c}{ }^{208} \mathrm{~Pb}-{ }^{208} \mathrm{~Pb}$ interactions. Results from the mixed events are shown (b).

on the basis of their $F_{2}$ values do have the densely populated phase space regions, $\eta$ and $\phi$ distributions of these events along with the "cold" event are plotted in Figure 3, whereas two-dimensional $\eta-\phi$ distributions for these events are exhibited in Figures 4 and 5. Moreover in order to ensure that the observed spikes and valleys are the event characteristics and are not due to statistical reasons $\eta$ and $\phi$ distributions of the corresponding five mixed events are also plotted in the same figures. It is interesting to note in these figures that onedimensional $\eta$ and $\phi$ distributions and two-dimensional $\eta-\phi$ 

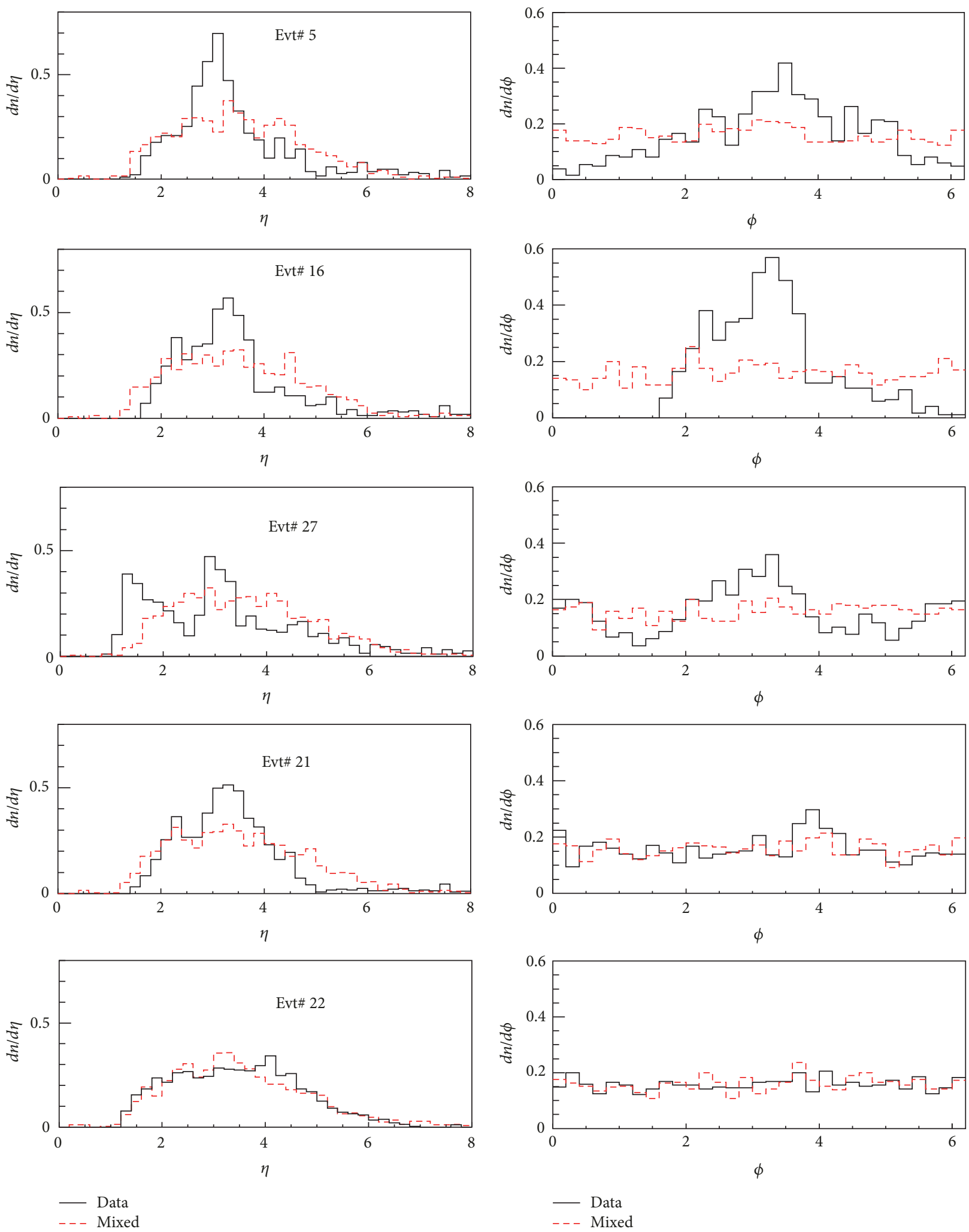

- Data

- - - Mixed

Figure 3: $\eta$ and $\phi$ distributions for the four "hot" and one "cold" $158 \mathrm{~A} \mathrm{GeV/c}{ }^{208} \mathrm{~Pb}-{ }^{208} \mathrm{~Pb}$ collision events (solid lines) and corresponding mixed events (broken lines). 

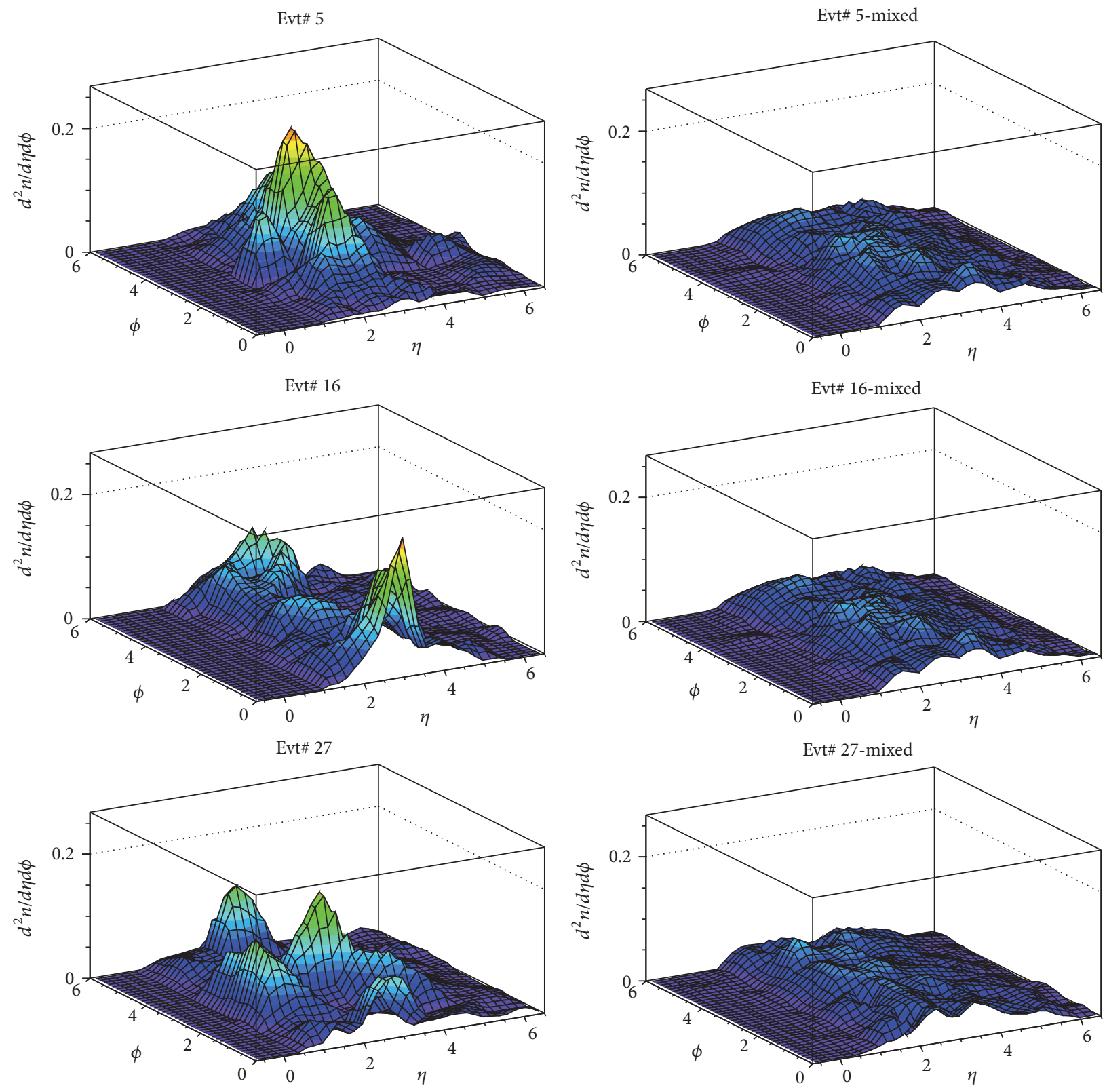

(a)

(b)

Figure 4: Two-dimensional $\eta-\phi$ distributions for events \# 5, 16, and 27 produced in $158 \mathrm{~A} \mathrm{GeV} / \mathrm{c}^{208} \mathrm{~Pb}^{208} \mathrm{~Pb}$ collisions (a) and corresponding mixed events (b).

distributions of the four "hot" events do exhibit distinct peaks and valleys while the fifth event (the "cold" event) shows no such spikes and the distributions of these events match with those obtained from the corresponding mixed events. It may also be noted from the figure that the particle densities in the spiky regions are larger than the expected average values by a factor of $\sim 2$.

2.3. Two-Particle Correlations. Studies involving multiparticle correlations are widely accepted as a tool to search for the occurrence of phase transition in relativistic AA collisions
$[42,43]$. The presence of the correlations among emitted particles guides us towards the fluctuation occurring in the observables during the phase transition. It has been reported $[43,44]$ that the inclusive two-particle correlations have two components: the direct two-particle correlations conventionally referred to as the short-range correlations (SRC) and the effective long-range correlations (LRC). The strong SRC have been reported to be present in several investigations [42-44]. These correlations have been reported to remain confined to a region, $\eta \pm 1$ units around mid-rapidity. Their properties have been explored by the concept of clustering 

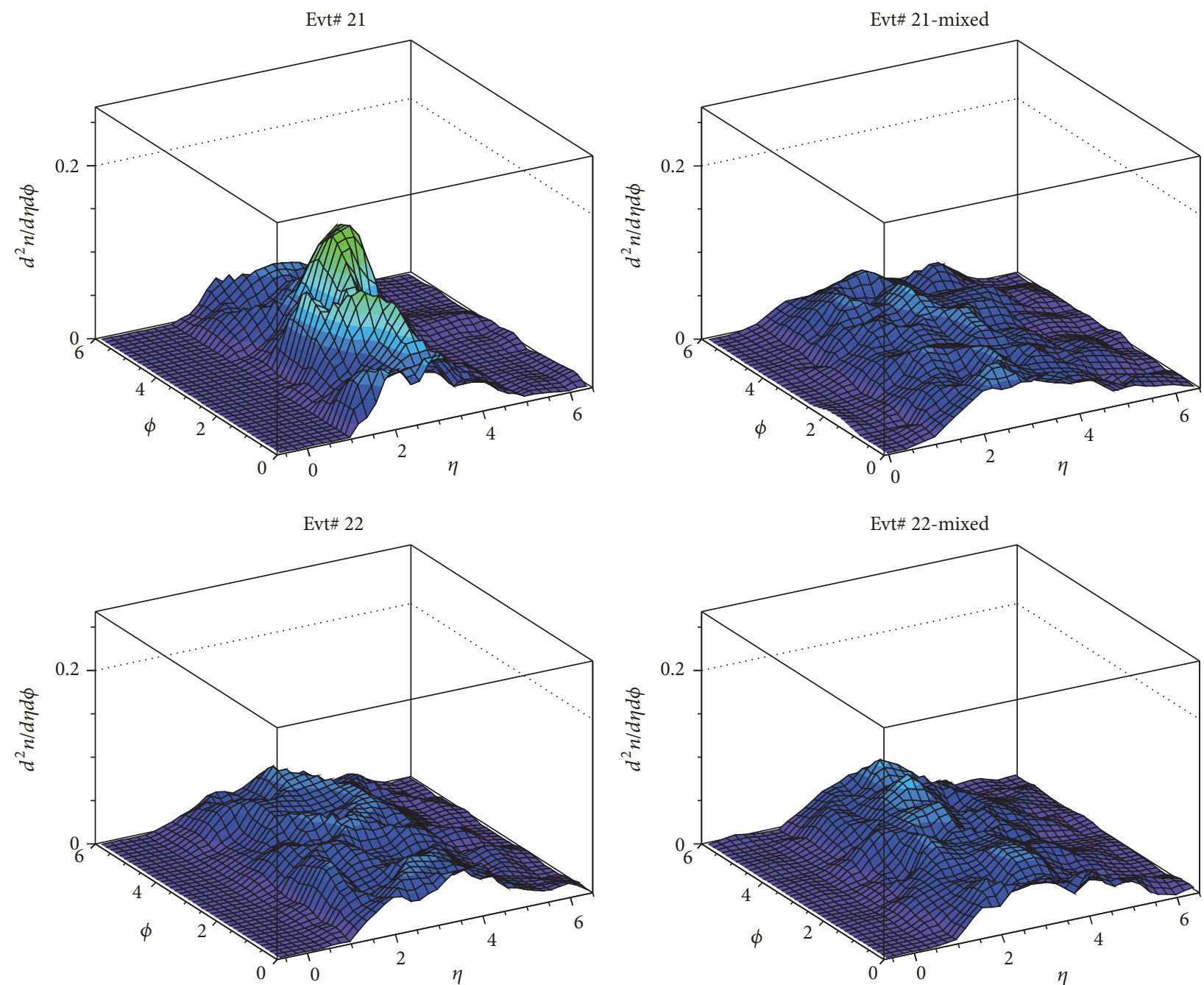

FIgure 5: The same plots as in Figure 4 but for Evts\# 21 and 22.

[44]. LRC, on the other hand, arise due to ebe fluctuations of overall multiplicity and are expected $[43,44]$ to extend over a relatively longer range ( $>2$ units of $\eta$ ). The idea of particle production through the formation of clusters, rather than the independent emission, has been widely adopted in describing the various features of particle production $[44,45]$. It is widely accepted that the SRC arise due to the tendency of hadrons to be grouped in clusters, which are formed during the intermediate stage of the collision. The clusters formed are independently emitted according to a dynamically generated distribution in $\eta$ and $\phi$ and then decay isotropically in their own rest frame into the real physical particles $[43,44]$ finally measured in the detectors. The observed two-particle correlations would permit disentangling different correlation sources which can be directly connected to the phenomena like collective flow, jets, resonance decays, and so forth [46].

Two-particle angular correlations in $\eta$ and $\phi$ spaces were first studied by ACM Collaboration at ISR energies [47]. It was observed that the dominant contribution to the correlation comes from the two- and three-body decay of resonances $\left(\eta, \rho^{0}, \omega\right)$. Two structures were discovered: (i) an enhancement near $\Delta \phi=\pi$ (away-side) explained by the two-body decay scenario and (ii) the enhancement at $\Delta \phi \simeq$ 0 together with an azimuthal ridge (centred at $\Delta \eta=0$ ) consistent with three-body decays. These features were, later on, confirmed by PHOBOS Collaboration [44]. Fluctuations observed in the physically measurable quantities provide us indirect measure of the width of the two particle densities. Thus, these are quite useful in providing us with additional information in comparison to those obtained by studying their averages. Besides this, a significant contribution to the two-particle correlations comes from the collective effects. They appear as a modulation in $\Delta \phi$ and are usually searched for in high multiplicity events $[46,48]$. An attempt is, therefore, made to study the two-particle correlations in individual events exhibiting large fluctuations in their $\eta$ and $\phi$ distributions which have been sorted out as discussed in the previous sections. The findings are compared with the corresponding mixed events which would help in extracting the contributions present due to the dynamical reasons. 

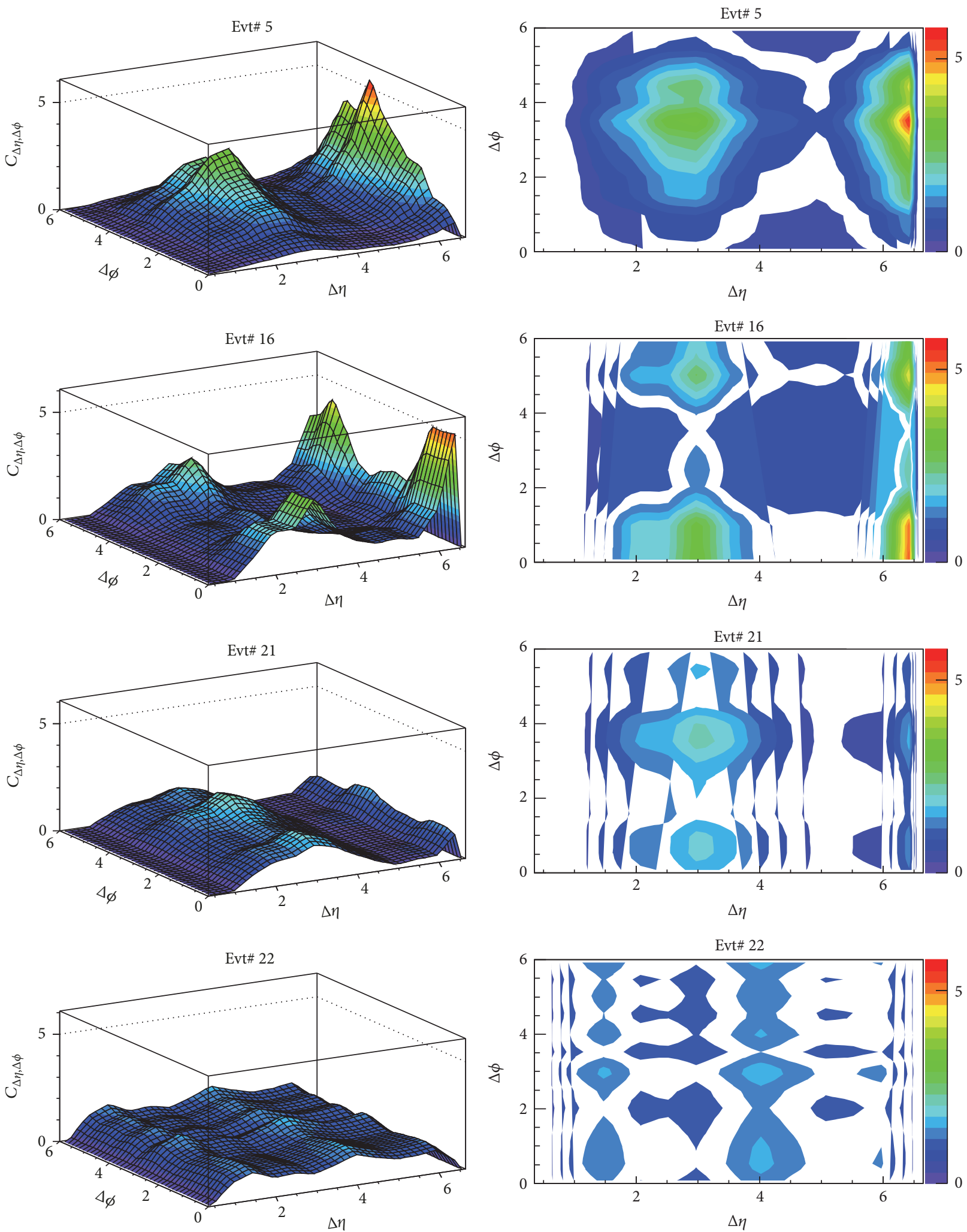

(a)

(b)

Figure 6: Correlation function $C(\Delta \eta, \Delta \phi)$ for Evts\# 5, 16, 21, and 22 produced in ${ }^{208} \mathrm{~Pb}^{2}{ }^{208} \mathrm{~Pb}$ collisions at $158 \mathrm{~A}$ GeV/c. The plots shown in (b) are the contour plots corresponding to those shown in (a). 

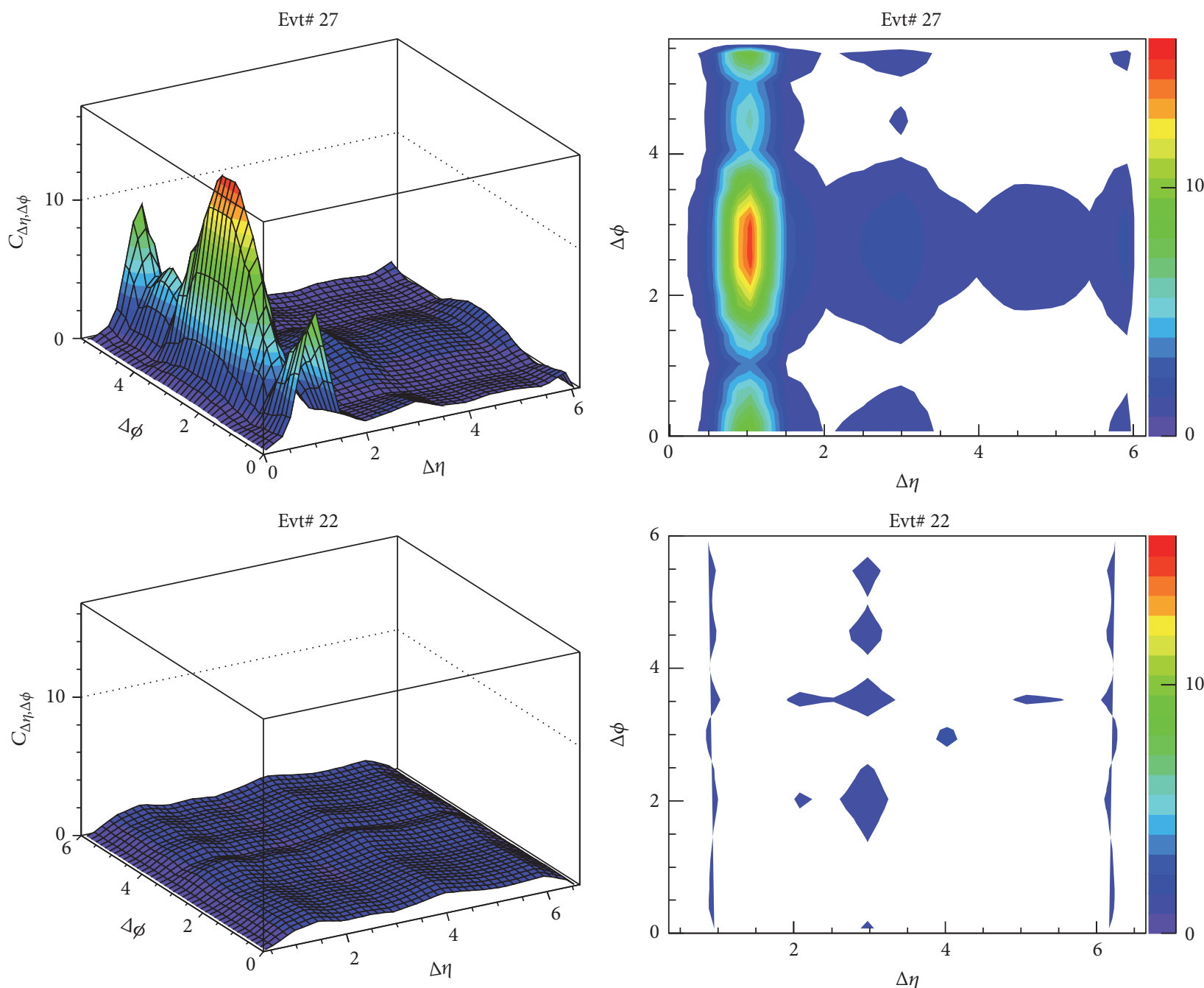

FIGURE 7: Same plot as in Figure 6 but for Evts\# 22 and 27: results from Evt\# 22, shown in Figure 6, are replotted here but on $z$-scale, different from that in Figure 6, for comparison with Evts\# 27.

Two-particle correlations are generally studied in terms of the differences in $\eta$ and $\phi$ values between the two particles produced in the same event [46].

$$
\begin{aligned}
& \Delta \eta=\left|\eta_{1}-\eta_{2}\right|, \\
& \Delta \phi=\left|\phi_{1}-\phi_{2}\right| .
\end{aligned}
$$

The correlation function $C(\Delta \eta, \Delta \phi)$ is defined and calculated as follows:

$$
\begin{aligned}
& C(\Delta \eta, \Delta \phi)=\frac{N_{\text {mixed }}^{\text {pair }} \rho_{d}^{\text {II }}(\Delta \eta, \Delta \phi)}{N_{\text {data }}^{\text {pair }} \rho_{m}^{\text {II }}(\Delta \eta, \Delta \phi)} \\
& \text { where, } \rho_{d}^{\text {II }}(\Delta \eta, \Delta \phi)=\frac{d^{2} N_{\text {data }}}{d \Delta \eta d \Delta \phi}, \rho_{m}^{\text {II }}=\frac{d^{2} N_{\text {mixed }}}{d \Delta \eta d \Delta \phi}
\end{aligned}
$$

being the distributions of pairs of particles from the data and mixed events, respectively. $\rho^{\mathrm{II}}(\Delta \eta, \Delta \phi)$ distributions for the data and mixed event were generated by counting the number of pairs in the intervals $\Delta \eta$ and $\Delta \phi$. For evaluating $C(\Delta \eta, \Delta \phi)$, the distributions for the data/mixed events were normalized to the number of pairs $\left(N_{\text {data }}^{\text {pair }}\right.$ or $\left.N_{\text {mixed }}^{\text {pair }}\right)$. The calculations are performed considering the charged particles of each event having $\phi$ values lying in the interval $0<\phi<2 \pi$ and $\eta$ values lying in the range $|\eta| \leq \eta_{c} \pm 3.0, \eta_{c}$ being the centre of symmetry of the $\eta$ distribution.

The correlation function $C(\Delta \eta, \Delta \phi)$ is estimated for the five events selected on the basis of the criteria discussed in the previous section and are plotted in Figures 6 and 7; the different scales of $z$-axis be noted for the plots for Evts\# 5 , 16, 21, and 22 shown in Figure 6 and that for Evts\# 27 and 22, displayed in Figure 7. The correlation function for Evt\# 22 (the "cold" event) is plotted in both the figures on different $Z$-scales of "hot" events for comparison's sake. The difference is noticeable due to the different $z$-scales chosen in the two figures. The $z$-scales in the two figures are kept the same as that for the other events in the figure so that the fluctuations in 
the hot and cold events can be clearly reflected. The following references may be drawn from Figures 6 and 7:

(1) In the "cold" event (Evt\# 22), shown in the bottom panel of the figures, presence of two-particle correlations is noticed in the region of $\Delta \eta\left(\eta_{c} \pm 2\right)$ and throughout the $\phi$ region considered. This indicates the presence of two-particle correlations in ${ }^{208} \mathrm{~Pb}$ ${ }^{208} \mathrm{~Pb}$ collisions at $158 \mathrm{~A} \mathrm{GeV} / \mathrm{c}$.

(2) Evt\# 21 shows few distinct peaks around $\eta_{c}(\sim 3.5)$ which spreads in considerable range of $\phi$. It is interesting to mention that the $F_{2}$ values of this event are significantly large in the $\eta$-space while in the $\phi$-space its $F_{2}$ values are not so large and compare well with the event averaged values (Figure 2).

(3) Evts\# 5 and 16 exhibit some distinct peaks at $\Delta \eta \sim$ 1.5 and 3.5 and 6.0. The magnitude of correlation, $C(\Delta \eta, \Delta \phi)$, in these regions is as large as $\sim 6$.

(4) Evt\# 27 at $\Delta \eta \sim 1.0$ gives very prominent peaks where the magnitude of correlations function is as high as $\sim 16$.

(5) The $F_{2}$ values of the Evts\# 5, 16, and 27 which exhibit strong two-particle correlations may also be noted to be quite large in $\eta, \phi$, and $\eta-\phi$ spaces. These events have been noticed to show dominant peaks and valleys in their $\eta$ and $\phi$ distributions too.

\section{Summary}

In the present article, we have studied the fluctuation observables relevant to investigation of ${ }^{208} \mathrm{~Pb}_{-}{ }^{208} \mathrm{~Pb}$ collisions at $158 \mathrm{~A} \mathrm{GeV} / \mathrm{c}$. Intermittency or factorial moment analysis applied to individual high multiplicity events may be used to identify events with densely populated narrow phase space regions. The four events selected on the basis of their $F_{2}$ values are found to exhibit large fluctuations in $\eta$ and $\phi$ distributions and exhibit strong two-particle correlations. The magnitude of the correlation function in few of the events appears to be as high as $\sim 16$. These observations give clear indication that if the system created during the collision has undergone phase transition, the associated fluctuation observables can be reliably analyzed on the basis of SFMs value. The result of the present analysis thus suggests that if the system formed during the collision has undergone the phase transition, the associated events identified on the basis of their SFMs values can be analyzed individually in detail which may lead to drawing some interesting conclusions.

\section{Conflicts of Interest}

The authors declare that there are no conflicts of interest.

\section{References}

[1] S. Shakeel Ahmad, M. M. Khan, S. Khan, A. Khatun, and M. Irfan, "A study of event-by-event fluctuations in relativistic heavy-ion collisions," International Journal of Modern Physics E, vol. 23, no. 11, Article ID 1450065, 18 pages, 2014.
[2] S. A. Voloshin, V. Koch, and H. G. Ritter, "Event-by-event fluctuations in collective quantities," Physical Review C: Nuclear Physics, vol. 60, no. 2, Article ID 024901, 1999.

[3] M. Döring and V. Koch, "Event-by-event fluctuations in heavy ion collisions," Acta Physica Polonica B, vol. 33, no. 6, pp. 14951504, 2002.

[4] L. D. Landau and E. M. Lifshitz, Statistical Physics, Pergamon Press, 1958.

[5] E. Shuryak, "Quark-gluon plasma-new frontiers," Journal of Physics G: Nuclear and Particle Physics, vol. 35, no. 10, Article ID 104044, 2008.

[6] H. van Hees, V. Greco, and R. Rapp, "Heavy-quark probes of the quark-gluon plasma and interpretation of recent data taken at the BNL Relativistic Heavy Ion Collider," Physical Review C: Nuclear Physics, vol. 73, no. 3, Article ID 034913, 2006.

[7] K. Adcox and PHENIX Collaboration, "Event-by-event fluctuations in mean $p_{T}$ and mean $e_{T}$ in $\sqrt{s_{N N}}=130 \mathrm{GeV} \mathrm{Au}+\mathrm{Au}$ collisions," Physical Review C: Nuclear Physics, vol. 66, no. 2, Article ID 024901, 2002.

[8] S. Ahmad, A. Ahmad, A. Chandra, M. Zafar, and M. Irfan, "Forward-backward multiplicity fluctuations in $200 \mathrm{~A} \mathrm{GeV} / \mathrm{c}$ ${ }^{16} \mathrm{O}-\mathrm{AgBr}$ and ${ }^{32} \mathrm{~S}-\mathrm{AgBr}$ collisions," Physica Scripta, vol. 87, no. 4, Article ID 045201, 2013.

[9] S. S. Adler and PHENIX Collaboration, "Measurement of nonrandom event-by-event fluctuations of average transverse momentum in $\sqrt{s_{N N}}=200 \mathrm{GeV} \mathrm{Au}+\mathrm{Au}$ and $p+p$ collisions," Physical Review Letters, vol. 93, no. 9, Article ID 092301, pp. 2229, 2004.

[10] D. Adamová, G. Agakichiev, H. Appelshäuser, V. Belaga, P. Braun-Munzinger et al., "Event-by-event fluctuations of the mean transverse momentum in 40,80 , and $158 \mathrm{~A} \mathrm{GeV} / \mathrm{c} \mathrm{Pb} \mathrm{Au}$ collisions," Nuclear Physics A, vol. 727, no. 1-2, pp. 97-119, 2003.

[11] F. Jinghua and L. Lianshou, "Extracting event dynamics from event-by-event analysis," Physical Review C, vol. 68, no. 6, Article ID 064904, 2003.

[12] L. Lianshou and F. Jinghua, "A Comparison of different measures for dynamical event-mean transverse momentum fluctuation," 2004, https://arxiv.org/abs/hep-ph/0401129.

[13] C. Alt, T. Anticic, and B. Baatar, "Energy dependence of $\phi$ meson production in central $\mathrm{Pb}+\mathrm{Pb}$ collisions at $\sqrt{s_{N N}}=6$ to $17 \mathrm{GeV}$," Physical Review C: Nuclear Physics, vol. 78, no. 4, Article ID 044907, 2008.

[14] S. Bhattacharyya, Phys. Rev. Lett. B, vol. 726, Article ID 1350066, 2013.

[15] M. L. Cherry, A. Dabrowska, P. Deines-Jones et al., "Event-byevent analysis of high multiplicity $\mathrm{Pb}$ (158-GeV/nucleon) $\mathrm{Ag} / \mathrm{Br}$ collisions," Acta Physica Polonica B, vol. 29, no. 8, pp. 2129-2146, 1998.

[16] S. Ahmad, A. Chandra, A. Kumar et al., "Event-by-event analysis of high-multiplicity events produced in $158 \mathrm{~A} \mathrm{GeV} / \mathrm{c}$ ${ }^{208} \mathrm{~Pb}-{ }^{208} \mathrm{~Pb}$ collisions," Europhysics Letters, vol. 112, no. 4, Article ID 42001, 2015.

[17] T. Anticic and NA49 Collaboration, "Search for the QCD critical point in nuclear collisions at $158 \mathrm{~A} \mathrm{GeV}$ at the CERN Super Proton Synchrotron (SPS)," Physical Review C, vol. 81, no. 6, 2010.

[18] A. Bialas and B. Ziaja, "Intermittency in a single event," Physics Letters B, vol. 378, no. 1-4, pp. 319-322, 1996.

[19] S. Mrowczynski, "Overview of event-by-event fluctuations," Acta Physica Polonica B, vol. 40, pp. 1053-1074, 2009. 
[20] L. Lianshou, "Single event 2-dimensional factorial moment analysis of ${ }^{197} \mathrm{Au}$-emulsion data from EMU01 experiment," Nuclear Physics B - Proceedings Supplements, vol. 71, no. 1-3, pp. 341-345, 1999.

[21] K. Fialkowski and R. Wit, "Entropy in cluster analysis of single events in heavy ion collisions," Acta Physica Polonica B, vol. 31, pp. 681-686, 2000.

[22] K. Fialkowski and R. Wit, "Event-by-event cluster analysis of final states from heavy ion collisions," Acta Physica Polonica B, vol. 30, pp. 2759-2765, 1999.

[23] M. Hauer, V. V. Begun, M. Gaździcki, M. I. Gorenstein, V. P. Konchakovski, and B. Lungwitz, "Multiplicity fluctuations in relativistic nuclear collisions: statistical model versus experimental data," Journal of Physics G: Nuclear and Particle Physics, vol. 35, no. 4, Article ID 044064, 2008.

[24] D. Ghosh, A. Deb, P. Mandal, S. Biswas, K. Chattopadhyay et al., "Non-statistical fluctuation of compound multiplicity in nucleus-nucleus interactions: evidence of strong intermittency," Chinese Physics Letters, vol. 19, no. 10, pp. 1436-1438, 2002.

[25] S. L. C. Barroso, A. O. deCarvalho, J. A. Chinellato et al., "Analysis of one hadron rich event," in Proceedings of the XVI International Symposium on Very High Energy Cosmic Ray Interactions, Batavia, Ill, USA, July 2010.

[26] Y. F. Wu and L. S. Liu, "Low average-transverse-momentum intermittency," Physics Letters B, vol. 269, p. 28, 1999.

[27] L. S. Liu, "Single event 2-dimensional factorial moment analysis of ${ }^{197} \mathrm{Au}$-emulsion data from EMU01 experiment," Nuclear Physics B - Proceedings Supplements, vol. 71, no. 1-3, pp. 341-345, 1999.

[28] M. I. Adamovich, M. M. Aggarwal, Y. A. Alexandrov et al., "Produced particle multiplicity dependence on centrality in nucleus - nucleus collisions," Journal of Physics G: Nuclear and Particle Physics, vol. 22, no. 10, p. 1469, 1996.

[29] M. I. Adamovich, M. M. Aggarwal, N. P. Andreeva et al., "Rapidity densities and their fluctuations in central $200 \mathrm{~A}$ $\mathrm{GeV}{ }^{32} \mathrm{~S}$ interactions with $\mathrm{Au}$ and $\mathrm{Ag}, \mathrm{Br}$ nuclei EMU01 collaboration," Physical Letters B, vol. 227, no. 2, pp. 285-290, 1989.

[30] M. I. Adamovich, M. M. Aggarwal, Y. A. Alexandrov et al., "Scaled-factorial-moment analysis of $200 \mathrm{~A}-\mathrm{GeV}$ sulfur + gold interactions," Physical Review Letters, vol. 65, article 412, 1990.

[31] M. I. Adamovich, Y. A. Alexandrov, and S. A. Asimov, "Multiplicities and rapidity densities in $200 \mathrm{AGeV}^{16} \mathrm{O}$ interactions with emulsion nuclei," Physics Letters B, vol. 201, no. 3, pp. 397402, 1988.

[32] M. I. Adamovich, M. M. Aggarwal, Y. A. Alexandrov et al., "On the systematic behaviour of the intermittency-indices in nuclear interactions," Physics Letters B, vol. 263, no. 3-4, pp. 539-543, 1991.

[33] A. Bialas and R. Peschanski, "Moments of rapidity distributions as a measure of short-range fluctuations in high-energy collisions," Nuclear Physics B, vol. 273, no. 3-4, pp. 703-718, 1986.

[34] V. Azhinenko, Y. A. Belokopytov, H. Böttcher et al., "Intermittency patterns in $\pi^{+} p$ and $K^{+} p$ collisions at $250 \mathrm{GeV} / \mathrm{c}$," Physics Letters B, vol. 222, no. 2, pp. 306-210, 1989.

[35] R. Holynski, A. Jurak, A. Olszewski, and B. Wilczynska, "Evidence for intermittent patterns of fluctuations in particle production in high-energy interactions in nuclear emulsion," Physical Review Letters, vol. 62, article 733, 1989.

[36] E. A. De Wolf, I. M. Dremin, and W. Kittel, "Scaling laws for density correlations and fluctuations in multiparticle dynamics," Physics Reports, vol. 270, no. 1-2, pp. 1-141, 1996.
[37] S. Ahmad, M. M. Khan, N. Ahmad, and A. Ahmad, "Erraticity behaviour in relativistic nucleus-nucleus collisions," Journal of Physics G: Nuclear and Particle Physics, vol. 30, no. 9, pp. 11451152, 2004.

[38] S. Ahmad, M. M. Khan, N. Ahmad, A. R. Khan, M. Zafar, and M. Irfan, "Erratic fluctuations in $14.5 \mathrm{~A} \mathrm{GeV} / \mathrm{c}^{28} \mathrm{Si}-\mathrm{AgBr}$ Collisions," Acta Physica Hungarica Series A, Heavy Ion Physics, vol. 25, no. 1, pp. 105-115, 2006.

[39] R. C. Hwa, "Beyond intermittency: erraticity" Acta Phys. Pol B, vol. 27, p. 1789, 1996.

[40] B. Wosiek, "Review of experimental results on intermittency," Acta Phys. Slov, vol. 46, no. 4, p. 531, 1996.

[41] A. Bialas and R. Peschanski, "Intermittency in multiparticle production at high energy," Nuclear Physics B, vol. 308, no. 4, pp. 857-867, 1988.

[42] B. I. Abelev, M. M. Aggarwal, Z. Ahammed et al., "Growth of long range forward-backward multiplicity correlations with centrality in $\mathrm{Au}+\mathrm{Au}$ Collisions at $\sqrt{s_{N N}}=200 \mathrm{GeV}$,' Physical Review Letters, vol. 103, Article ID 172301, 2009.

[43] S. Ahmad, A. Chandra, M. Zafar et al., "Short- and long-range multiplicity correlations in relativistic heavy-ion collisions," International Journal of Modern Physics E, vol. 22, no. 9, Article ID 1350066, 2013.

[44] B. Alver, B. B. Back, M. D. Baker et al., "Cluster properties from two-particle angular correlations in $p+p$ collisions at $\sqrt{s}=200$ and $410 \mathrm{GeV}$," Physical Review C, vol. 75, Article ID 054913, 2007.

[45] UA5 Collaboration, R. E. Ansorge, B. Åsman, C. N. Booth, L. Burow et al., "Charged particle correlations in $\bar{p} p$ collisions at c.m. energies of 200, 546 and 900 GeV," Zeitschrift für Physik C Particles and Fields, vol. 37, no. 2, pp. 191-213, 1988.

[46] A. Aduszkiewicz, Y. Ali, E. Andronov, T. Antićić, N. Antoniou et al., "Two-particle correlations in azimuthal angle and pseudorapidity in inelastic $\mathrm{p}+\mathrm{p}$ interactions at the CERN Super Proton Synchrotron," The European Physical Journal C, vol. 77, article 59, 2017.

[47] K. Eggert, H. Frenzel, W. Thomé et al., "Angular correlations between the charged particles produced in pp collisions at ISR energies," Nuclear Physics B, vol. 86, no. 2, pp. 201-215, 1975.

[48] G. Agakishiev, M. M. Aggarwal, Z. Ahammed et al., "Anomalous centrality evolution of two-particle angular correlations from Au-Au collisions at $\sqrt{s_{N N}}=62$ and $200 \mathrm{GeV}$,' Physical Review C: Nuclear Physics, vol. 86, Article ID 064902, 2012. 

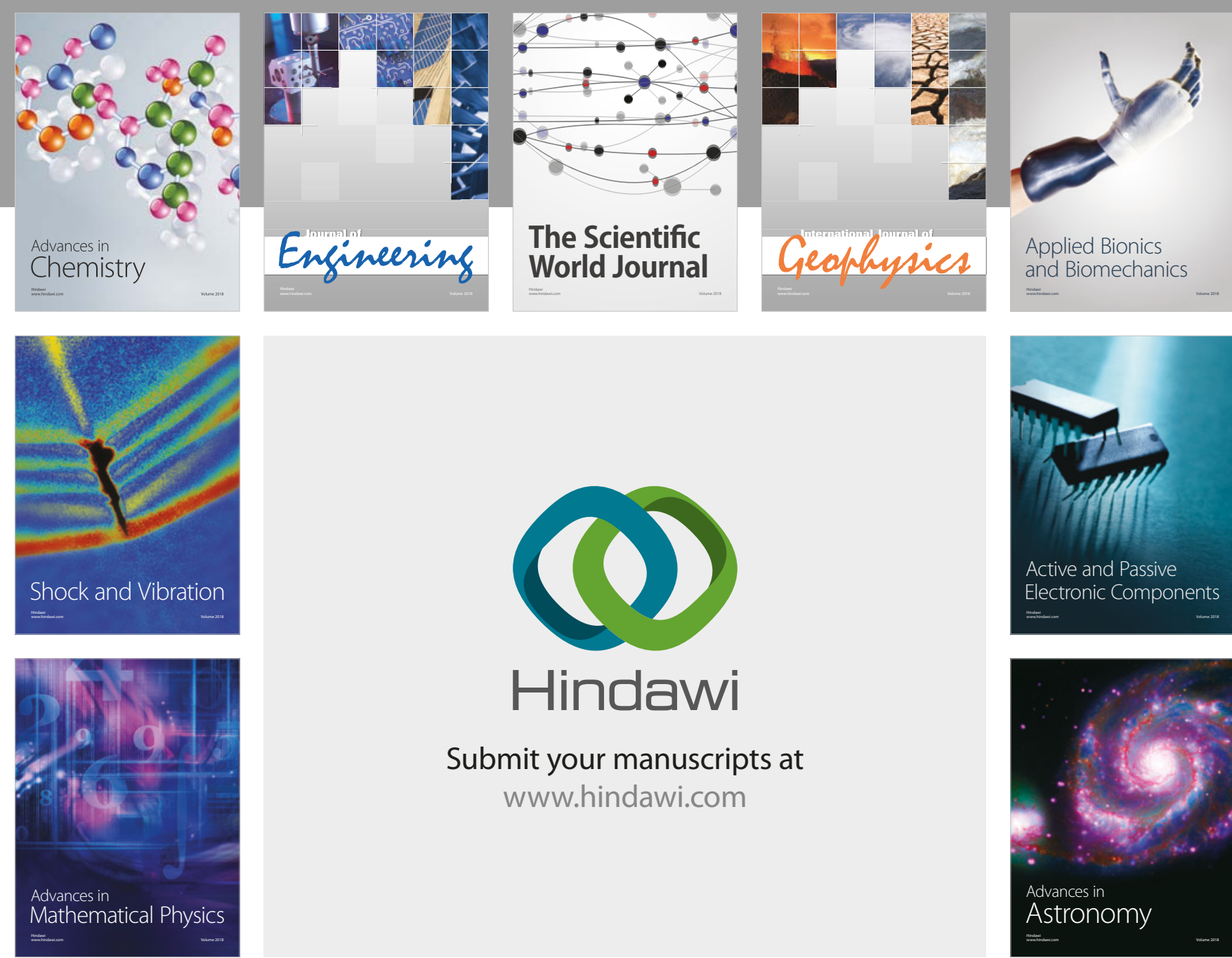

Submit your manuscripts at

www.hindawi.com

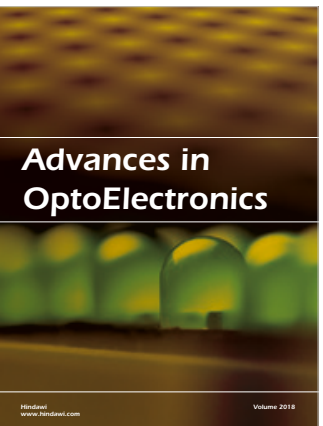

\section{Rotcting Machinery}
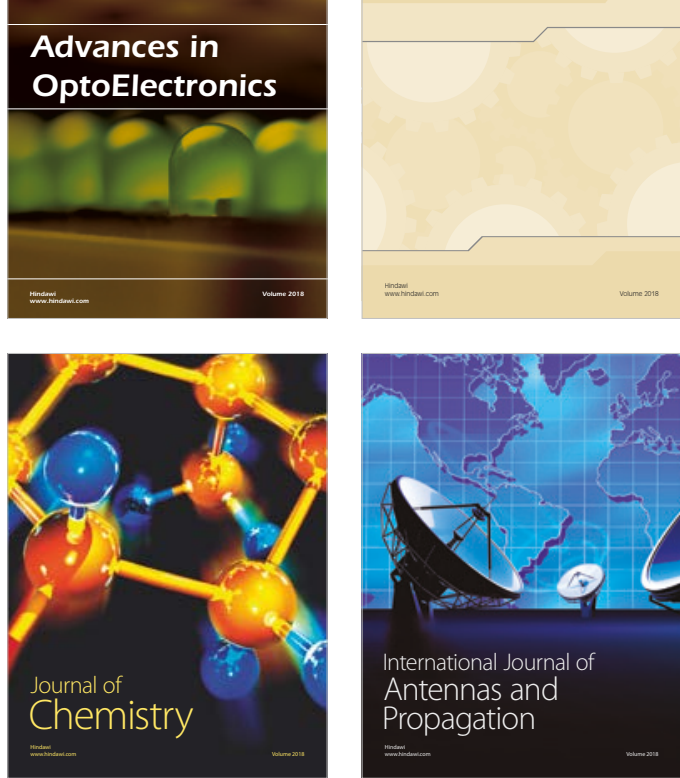

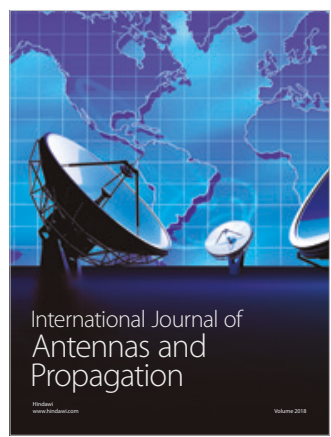

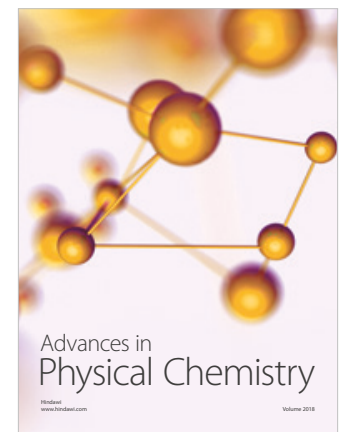

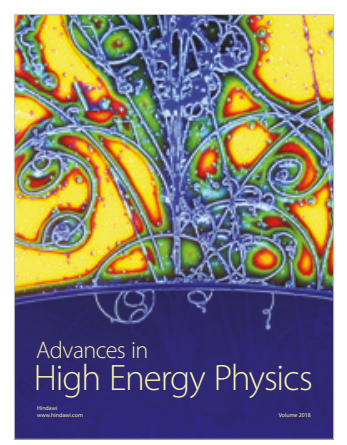

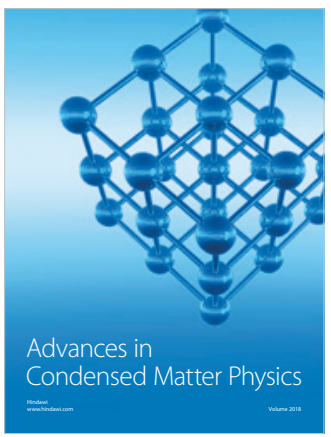

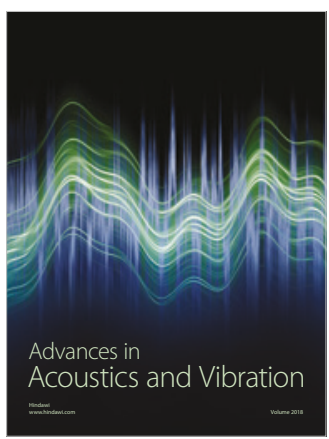

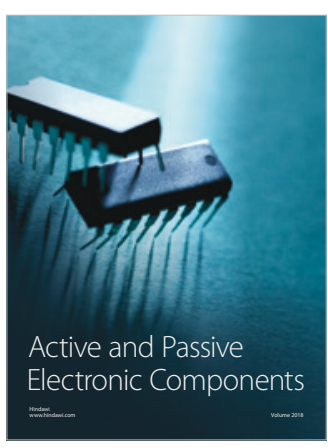
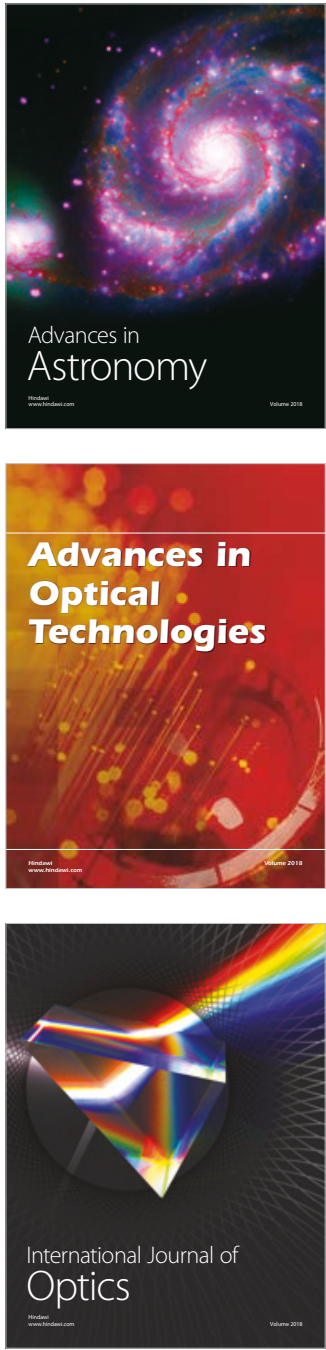